\title{
Elucidating fuel cell catalyst degradation mechanisms by identical-location transmission electron microscopy
}

Haoran $\mathrm{Yu}^{1}$, Michael Zachman ${ }^{2}$, Deborah Myers ${ }^{3}$, Rangachary Mukundan ${ }^{4}$, Hanguang Zhang ${ }^{5}$, Piotr Zelenay $^{5}$, Kenneth Neyerlin ${ }^{6}$ and David Cullen ${ }^{2}$

${ }^{1}$ Center for Nanophase Materials Sciences, Oak Ridge National Laboratory, Oak Ridge, Tennessee, United States, ${ }^{2}$ Center for Nanophase Materials Sciences, Oak Ridge National Laboratory, Tennessee, United States, ${ }^{3}$ Chemical Sciences and Engineering Division, Argonne National Laboratory, United States, ${ }^{4}$ Los Alamos National Laboratory, United States, ${ }^{5}$ Materials Physics and Applications Division, Los Alamos National Laboratory, United States, ${ }^{6}$ Chemistry and Nanoscience Center, National Renewable Energy Laboratory, United States

The recent surge in interest in hydrogen and fuel cells for heavy duty vehicle applications has put increased focus on improving the durability of both state-of-the-art Pt alloy nanoparticle and atomically-dispersed platinum group metal-free (PGM-free) oxygen reduction reaction catalysts [1,2]. Microscopy analysis of catalyst degradation usually utilizes a thin slice of pre- and post-mortem membrane electrode assemblies (MEAs) prepare by ultramicrotome. However, due to inhomogeneities in the MEA catalyst layers, it can be challenging to pinpoint the precise degradation mechanisms that lead to performance loss.

To address this limitation, electron microscopy methods have been developed that enable characterization of the same catalyst particles at various stages in the life cycle of a material. For example, in situ liquid electrochemical cells have been designed to analyze catalyst degradation during potential cycling within the transmission electron microscope (TEM) [3]. However, interactions between the liquid electrolyte and electron beam reduce resolution and may introduce artefacts. Identical-location transmission electron microscopy (IL-TEM), on the other hand, is a method that allows the same regions of a material to be studied before and after ex situ cycling without loss of spatial resolution [4,5]. For IL-TEM experiments, TEM grids (usually made of gold) are used to support catalysts that are subjected to electrochemical cycling in a three-electrode cell, as shown in Figure 1a. Changes in catalyst properties, namely particle size distribution, composition, and crystalline structure, can then be directly characterized before and after cycling. Since the specimen is identical to a conventional TEM specimen, imaging and analytical methods like energy-dispersive X-ray spectroscopy and electron energy-loss spectroscopy can be performed at high resolution.

IL-TEM has previously been used to elucidate Pt catalyst degradation mechanisms such as migration, coalescence, and detachment from the carbon support [6-8]. Ostwald ripening has not been observed in most IL-TEM studies [6-8], in stark contrast to MEA tests in which it is identified the dominant degradation mechanism [9]. Reproducing processes that occur in MEAs is critical to accurately identifying degradation mechanisms that can inform mitigation strategies.

In this work, we seek to identify the IL-TEM experimental conditions and protocols (Figure 1b) which most closely mimic end of life (EOL) morphology and composition found in MEAs. To reproduce the catalyst degradation observed in both Pt-based and PGM-free MEAs, a range of parameters are being explored such as specimen preparation method, electrolyte concentration, gaseous environment, upper and lower cycling potential, and potential wave shape. Figure 2 shows how different classes of fuel cell catalysts behave under the conditions showing in Figure 1. For a Pt/C catalyst (Figure 2a, b), significant changes in particle size were observed in high-angle annular dark-field scanning transmission electron microscopy (HAADF-STEM) images, with the complete loss of particles below $2 \mathrm{~nm}$. However, for a 
$\mathrm{PtCo} / \mathrm{C}$ alloy catalyst, very little change in particle size and composition was observed (Figure $2 \mathrm{c}$, $\mathrm{d}$ ), in stark contrast to MEA tests [10]. For the PGM-free system, degradation was found in the form of severe Fe loss (Figure 2e, f). We will present the direct comparison between IL-TEM and MEA tests for each class of catalysts and discuss how sample preparation and cycling parameters can be adjusted to replicate real-life losses more closely. The contribution of different degradation mechanisms revealed by IL-TEM in each system will then be presented, including particle coalescence, Ostwald ripening, transition metal leaching, and carbon corrosion. Accurate insights into fundamental fuel cell catalyst degradation mechanisms will then be used to guide development of catalysts with enhanced properties [11].

This manuscript has been authored by UT-Battelle, LLC under Contract No. DE-AC05-00OR22725 with the U.S. Department of Energy. The United States Government retains and the publisher, by accepting the article for publication, acknowledges that the United States Government retains a non-exclusive, paidup, irrevocable, world-wide license to publish or reproduce the published form of this manuscript, or allow others to do so, for United States Government purposes. The Department of Energy will provide public access to these results of federally sponsored research in accordance with the DOE Public Access Plan (http://energy.gov/downloads/doe-public-access-plan).

a)

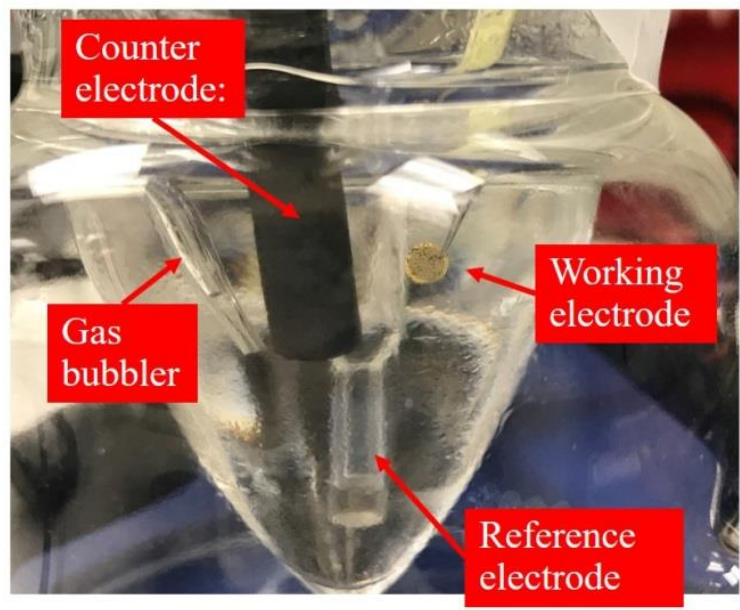

b)

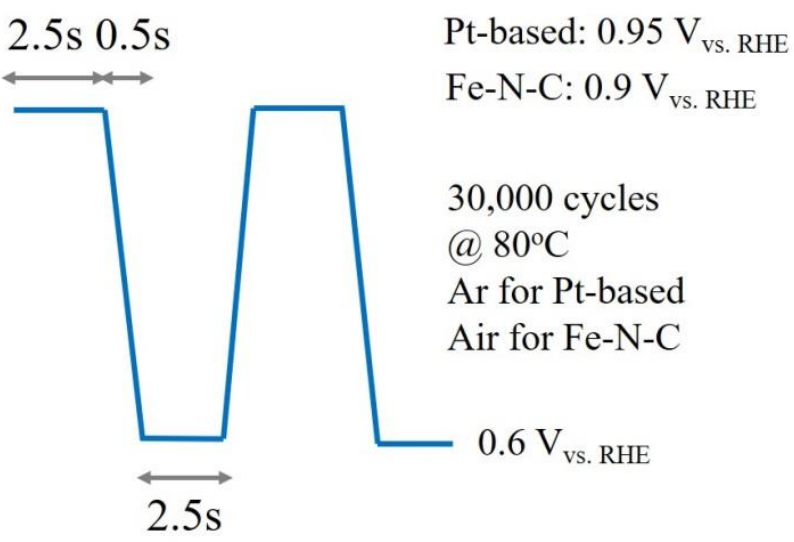

Figure 1. Figure 1. (a) Electrochemical cell setup for IL-TEM experiments. (b) Accelerated stress test and conditions used for experiments presented in Figure 2. 


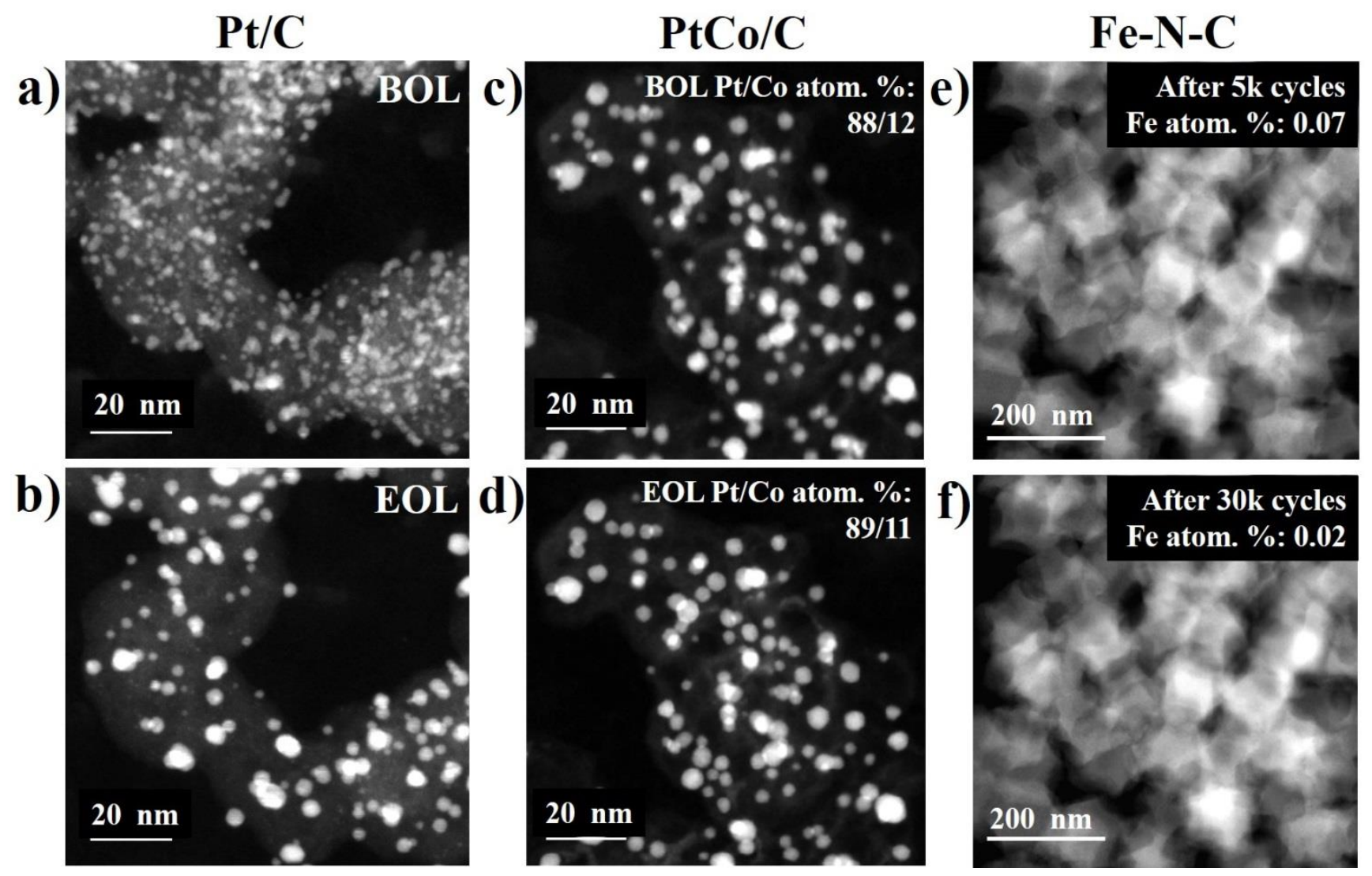

Figure 2. Figure 2. HAADF-STEM images acquired at beginning of life (BOL) and EOL for (a,b) Pt/C catalyst, (c,d) $\mathrm{PtCo} / \mathrm{C}$ alloy catalyst, and (e,f) PGM-free $\mathrm{Fe}-\mathrm{N}-\mathrm{C}$ catalyst. Inset compositional quantification of these regions determined by energy dispersive X-ray spectroscopy.

References

[1] RL Borup et al., Curr. Opin. Electrochem. 21 (2020), p. 192.

[2] L Osmieri et al., Curr. Opin. Electrochem. 25 (2021), p. 100627.

[3] V Beermann et al., Energy Environ. Sci. 12 (2019), p. 2476.

[4] KJJ Mayrhofer et al., J. Power Sources 185 (2008), p. 734.

[5] RM Aran-Ais et al., J. Am. Chem. Soc. 137 (2015), p. 14992.

[6] K Schlogl et al., J. Electroanal. Chem. 662 (2011), p. 355.

[7] Y Yu et al., Nano Lett. 12 (2012), p. 4417.

[8] L Dubau, L et al., Electrochim. Acta 110 (2013), p. 273.

[9] H Yu, H et al., Electrochim. Acta 247 (2017), p. 1169.

[10] TE O’Brien et al., ECS Trans. 98 (2020), p. 505.

[11] This work was supported by the U.S. Department of Energy (DOE), Energy Efficiency and Renewable Energy, Fuel Cell Technologies Office under the M2FCT and ElectroCat 2.0 consortia. Electron microscopy was conducted at the Center for Nanophase Materials Sciences, which is a DOE Office of Science User Facility. 